\title{
In Situ Nanoscale Investigation of Catalytic Reactions in the Liquid Phase Using Zirconia-Protected Tip-Enhanced Raman Spectroscopy Probes
}

\author{
Naresh Kumar, ${ }^{\dagger, \dagger, \S(0)}$ Caterina S. Wondergem, ${ }^{\dagger, \S}$ Andrew J. Wain, ${ }^{\ddagger \odot}$ and Bert M. Weckhuysen* ${ }^{\dagger} \uparrow$ \\ ${ }^{\dagger}$ Inorganic Chemistry and Catalysis Group, Debye Institute for Nanomaterials Science, Utrecht University, Universiteitsweg 99, \\ 3584 CG Utrecht, The Netherlands \\ ${ }^{\ddagger}$ National Physical Laboratory, Hampton Road, Teddington, TW11 0LW, United Kingdom
}

Supporting Information

\begin{abstract}
Tip-enhanced Raman spectroscopy (TERS) is a promising technique that enables nondestructive and label-free topographical and chemical imaging at the nanoscale. However, its scope for in situ characterization of catalytic reactions in the liquid phase has remained limited due to the lack of durable and chemically inert plasmonically active TERS probes. Herein, we present novel zirconia-protected TERS probes with 3 orders of magnitude increase in lifetime under ambient conditions compared to unprotected silvercoated probes, together with high stability in liquid media. Employing the plasmon-assisted oxidation of $p$-aminothiophenol as a model reaction, we demonstrate that the highly robust, durable, and chemically inert zirconiaprotected TERS probes can be successfully used for nanoscale spatially resolved characterization of a photocatalytic reaction within an aqueous environment. The reported improved lifetime and stability of probes in a liquid environment extend the potential scope of TERS as a nanoanalytical tool not only to heterogeneous catalysis but also to a range of scientific disciplines in which dynamic solid-liquid interfaces play a defining role.
\end{abstract}

$\mathrm{T}$ he rational design of novel, chemically functional nanomaterials with tailored properties relies on a deep understanding of structure-behavior relationships. This is especially poignant in the case of heterogeneous catalysis, in which the identification of active sites at spatially nonuniform solid-liquid and solid-gas interfaces under dynamic reaction conditions is key to materials and process optimization. ${ }^{1,2}$ However, conventional analytical techniques, such as Raman, infrared (IR), ultraviolet-visible, and fluorescence spectroscopies, often lack the required sensitivity and spatial resolution to achieve this ambitious goal. ${ }^{3,4}$ In recent years, tip-enhanced Raman spectroscopy (TERS) (Figure 1) has emerged as a potential solution to this challenge, enabling nondestructive topographical and molecular imaging of surfaces at the nanoscale. ${ }^{5,6}$

In TERS, a metallic scanning probe microscopy (SPM) probe, positioned within the excitation laser spot of a Raman microscope (Figure 1), enhances the incident electric field by several orders of magnitude via a combination of localized surface plasmon resonance (LSPR) and lightening rod effects, confining the field enhancement to a region similar in size to the probe apex. This near-field approach significantly improves the sensitivity of Raman microscopy and pushes its spatial resolution far beyond the diffraction limit. This technique has been successfully used in a wide range of research areas including biology, ${ }^{7}$ polymer blends, ${ }^{8}$ semiconductors, ${ }^{9}$ crystal- line materials, ${ }^{10}$ solar energy conversion, ${ }^{11}$ nanomaterials research, ${ }^{12-16}$ and single-molecule imaging. ${ }^{17,18}$

Despite the advantages of high spatial resolution and rich chemical information offered by TERS, ${ }^{1,19,20}$ only a handful of TERS studies of catalytic reactions have been reported so far. ${ }^{21-28}$ Furthermore, the majority of such studies have been carried out in ambient air or ultrahigh vacuum, with only a few in liquid environments, mostly limited to point spectroscopy measurements. ${ }^{29-34}$ 2D nanoscale chemical imaging of catalytic reactions in liquids represents a key challenge but has not been achieved using TERS, primarily due to the chemical reactivity, ${ }^{22,35}$ short lifetime, ${ }^{36-39}$ and/or instability of TERS probes in liquids. ${ }^{29,34}$ The chemical reactivity of metallic TERS probes is a particularly important consideration when studying catalysis in situ due to the potential for interference in the reaction under investigation. To address this issue, as well as extend the lifetime of metallic TERS probes, the use of an ultrathin coating of dielectric material such as $\mathrm{Al}_{2} \mathrm{O}_{3}{ }^{23,40}$ or $\mathrm{SiO}_{2}{ }^{41}$ as a protective layer has been reported. However, $\mathrm{Al}_{2} \mathrm{O}_{3}$ and $\mathrm{SiO}_{2}$ are not stable over the entire $\mathrm{pH}$ range, ${ }^{42}$ limiting the conditions in which the TERS probes could be used. Furthermore, both $\mathrm{Al}_{2} \mathrm{O}_{3}$ and $\mathrm{SiO}_{2}$

Received: January 16, 2019

Accepted: March 22, 2019

Published: March 27, 2019 


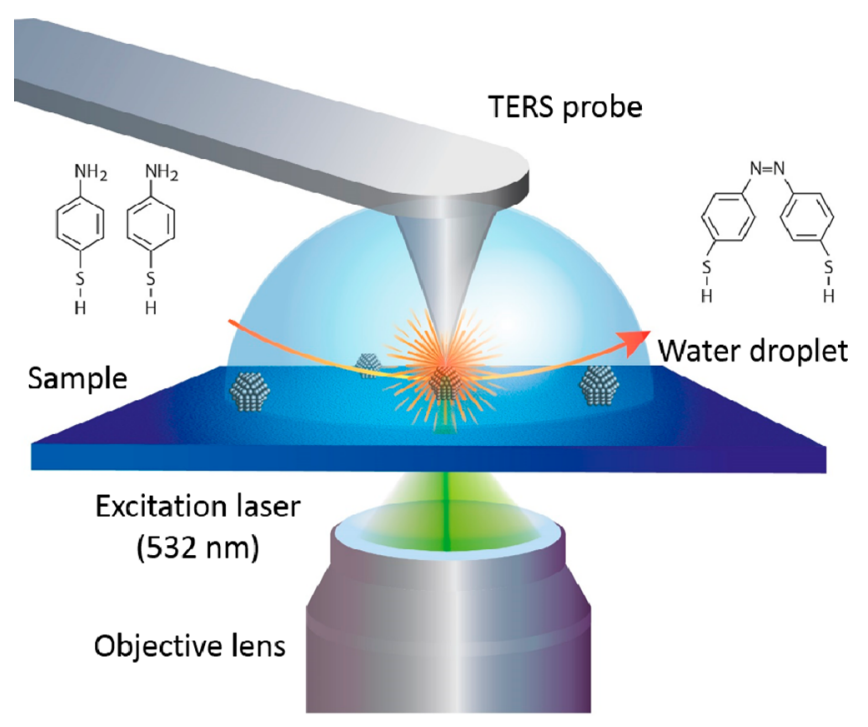

Figure 1. Schematic of the experimental TERS setup used in this work for spatially resolved mapping of plasmon-assisted oxidation of $p$-aminothiophenol ( $\mathrm{pATP})$ to $p, p^{\prime}$-dimercaptoazobenzene (DMAB) over a heterogeneous $\mathrm{Ag}$ substrate in an aqueous environment.

coatings only increased the lifetime of TERS probes for approximately 1 month, and although some increased stability in liquids was observed for the $\mathrm{Al}_{2} \mathrm{O}_{3}$-protected probes, ${ }^{30}$ no TERS mapping was performed. Recently, a more effective method of protecting TERS probes using atomic layer deposition (ALD) has been reported by Huang et al., where the authors presented three different coatings, $\mathrm{SiO}_{2}, \mathrm{Al}_{2} \mathrm{O}_{3}$, and $\mathrm{TiO}_{2}$, and successfully showed that the $\mathrm{SiO}_{2}$-coated STMTERS probes could enhance the Raman signal from a selfassembled monolayer of thiol molecules while suppressing the signal from pyridine molecules present in solution. ${ }^{43}$ However, TERS data was presented only for the $\mathrm{SiO}_{2}$-coated probes, and the mechanical stability, long-term stability of plasmonic signal enhancement, and capability of $2 \mathrm{D}$ chemical imaging in a liquid environment was not demonstrated. Furthermore, the use of ALD to produce ultrathin dielectric coatings requires expensive experimental apparatus and time-consuming optimization of deposition conditions.

Herein, we present novel atomic force microscopy (AFM)TERS probes with a multilayer metal coating protected using an ultrathin layer of zirconia $\left(\mathrm{ZrO}_{2}\right)$ that successfully overcome the key limitations of short lifetime, chemical inertness, and instability in a liquid environment. $\mathrm{ZrO}_{2}$ offers excellent catalytic support properties for several reactions ${ }^{44,45}$ and stability over the entire $\mathrm{pH}$ range, ${ }^{42}$ making it especially suitable for investigating heterogeneous catalytic reactions under a wide range of conditions. Compared to ALD, the simple wet-chemical method of $\mathrm{ZrO}_{2}$ coating developed in this work is far cheaper and could be easily implemented in any laboratory fume cupboard. Furthermore, using the novel TERS probes, we demonstrate the feasibility of mapping of a catalytic reaction over a heterogeneous metal surface in water with nanoscale spatial resolution, employing the photocatalytic oxidation of $p$-aminothiophenol (pATP) to $p, p^{\prime}$-dimercaptoazobenzene (DMAB) as a model reaction (Figure 1). ${ }^{23,46}$

Extending the Plasmonic Lifetime of TERS Probes. Experimental details are presented in Supporting Information (SI) section S1, including development of the $\mathrm{ZrO}_{2}$ coating procedure (Figures S1-S4). TEM measurements confirmed that the procedure was successful in coating a layer of $\mathrm{ZrO}_{2}$ on Ag-coated TERS probes with a thickness of 1-5 nm (Figure S5). Note that the TEM measurement was conducted after using the probe for continuous TERS measurements in water for $\sim 2 \mathrm{~h}$, which demonstrates the stability of the $\mathrm{ZrO}_{2}$ coating. The $\mathrm{ZrO}_{2}$ coating was also found to be stable in an aqueous environment for a long period of time and over the entire $\mathrm{pH}$ range (see Figure S3 and S4).

The lifetime of $\mathrm{ZrO}_{2}$-protected TERS probes was compared with that of the unprotected (Ag-coated) TERS probes using a poly(3,4-ethylenedioxythiophene)-poly(styrenesulfonate) (PEDOT:PSS) thin film as a test sample. Plasmonic enhancement of a TERS probe can be monitored using the ratio of the Raman signal intensity in the near-field (plasmonically enhanced electric field at a TERS probe apex) and far-field (electric field in the entire excitation laser spot), which is known as "contrast" and defined as follows

$$
\text { Contrast }=\frac{I_{\mathrm{TERS}}}{I_{\mathrm{FF}}}-1
$$

where $I_{\mathrm{TERS}}$ and $I_{\mathrm{FF}}$ are the intensities of a Raman band with the TERS probe in contact with and retracted from the sample, respectively. Time series TERS spectra recorded using unprotected and $\mathrm{ZrO}_{2}$-protected TERS probes stored in ambient air are shown in Figure 2a,b, respectively. Additional time series spectra recorded using the unprotected and $\mathrm{ZrO}_{2}-$ protected TERS probes are presented in Figures S6 and S7. Contrast was determined using the intensity of the $1454 \mathrm{~cm}^{-1}$ PEDOT:PSS band $\left(\nu_{\text {sym }, \mathrm{C} \alpha=\mathrm{C} \beta}\right)$ and is plotted in Figure $2 \mathrm{c}, \mathrm{d}$ for the unprotected and protected probes, respectively. The near-field signal intensity of a fresh unprotected TERS probe is found to be almost $3 \times$ higher than the far-field signal, indicating a strong plasmonic enhancement of the electric field at the probe apex. However, the TERS contrast decreases rapidly by $50 \%$ of its initial value (half-life) within $4.5 \mathrm{~h}$ due to surface oxidation of the Ag coating in air. ${ }^{36}$ After a rapid initial decline, the contrast decreases rather gradually by $75 \%$ in $10 \mathrm{~h}$, eventually reaching 0 after $170 \mathrm{~h}$ of exposure to air, indicating a complete loss of plasmonic enhancement. On the other hand, although the $\mathrm{ZrO}_{2}$-protected TERS probes exhibit a decrease in contrast of almost $50 \%$ compared to the unprotected probes (after 2 days of preparation), they show a much higher plasmonic stability; even after 140 days of exposure to the ambient environment, the TERS contrast decreased by only $43 \%$, indicating that the plasmonic sensitivity for TERS measurements is largely preserved. The pinhole-free $\mathrm{ZrO}_{2}$ layer likely blocks contact of the $\mathrm{Ag}$ coating with oxygen and moisture present in the surroundings, thereby preventing plasmonic degradation. ${ }^{36}$ From extrapolation of the time series plot in Figure $2 \mathrm{~d}$, the half-life of the $\mathrm{ZrO}_{2}$-protected TERS probes is estimated to be over 160 days. This corresponds to more than an $850 \times$ increase in lifetime compared to unprotected probes and to the best of our knowledge is the longest lifetime of any TERS probe stored under ambient conditions reported to date. Furthermore, unlike STM-based TERS, these novel $\mathrm{ZrO}_{2}$-protected probes could be employed for nanoscale chemical mapping of a wide range of conducting and nonconducting sample surfaces using an AFM-TERS setup.

Nanoscale Mapping of a Photocatalytic Reaction in a Liquid Environment. We next used $\mathrm{ZrO}_{2}$-protected TERS probes to demonstrate spatially resolved nanoscale characterization of a 

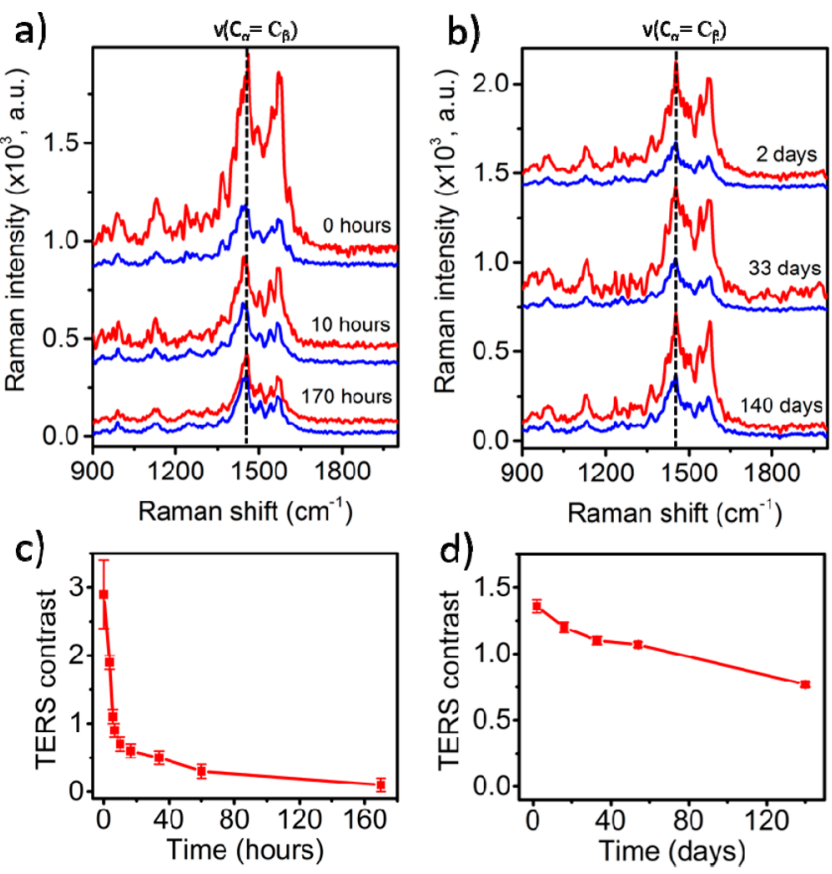

Figure 2. Time series TERS (red) and far-field Raman (blue) spectra measured from a PEDOT:PSS thin film on glass after exposing (a) unprotected TERS probes for 0,10 , and $170 \mathrm{~h}$ and (b) $\mathrm{ZrO}_{2}$ protected TERS probes for 2, 33, and 140 days to the ambient environment. Integration time: $30 \mathrm{~s}$. Laser power: $50 \mu \mathrm{W}$. Plots of TERS contrast versus exposure time of representative TERS probes to the ambient environment for measurements presented in (c) Figures $2 \mathrm{a}$ and $\mathrm{S} 6$ and (d) Figures $2 \mathrm{~b}$ and S7. In this study, a thin film of PEDOT:PSS was chosen to measure the lifetime of the zirconiacoated TERS tips because of its high chemical stability, low surface roughness, and strong Raman signal. ${ }^{30,36,40,47,48}$ For example, the root-mean-square surface roughness of a spin-coated PEDOT:PSS film has been shown to be $1.2 \pm 0.1 \mathrm{~nm}$ using tapping mode AFM, confirming a smooth topography. ${ }^{49}$ In order to further minimize the effect of surface roughness, the time series contrast plotted in Figure $2 \mathrm{c}, \mathrm{d}$ was measured from an average of three TERS and far-field measurements conducted at different areas on the PEDOT:PSS thin film.

catalytic reaction within an aqueous environment. We selected the photocatalytic oxidation of pATP $\rightarrow$ DMAB as a model reaction due to the ease of monitoring using the distinct Raman bands of the azo group of DMAB, appearing in the $1140-1500 \mathrm{~cm}^{-1}$ spectral region. ${ }^{46,50}$ pATP $\rightarrow$ DMAB is a well-understood and well-characterized plasmon-assisted reaction wherein hot electrons generated via LSPR of metal nanoparticles cause dissociation of adsorbed oxygen under ambient conditions, facilitating oxidative dimerization. ${ }^{51}$ On a plasmonic metal substrate, sites exhibiting a stronger LSPR are expected to act as more active catalysts. Therefore, we used a self-assembled monolayer (SAM) of pATP molecules on a heterogeneous $\mathrm{Ag}$ substrate containing nanostructures varying from 1 to $9 \mathrm{~nm}$ in height (Figure S8) as the test sample to map pATP $\rightarrow$ DMAB within a liquid environment using TERS. The Ag substrate was surface-enhanced Raman spectroscopy (SERS)-active with the $532 \mathrm{~nm}$ excitation laser. Characteristic $a_{\mathrm{g}}$ Raman bands of DMAB at $1142 \mathrm{~cm}^{-1}\left(\beta_{\mathrm{C}-\mathrm{H}}\right), 1390 \mathrm{~cm}^{-1}$ $\left(\nu_{\mathrm{N}=\mathrm{N}}\right)$, and $1437 \mathrm{~cm}^{-1}\left(\nu_{\mathrm{N}=\mathrm{N}}\right)$ could be clearly observed in the SERS spectrum of pATP adsorbed on the Ag substrate, indicating conversion to DMAB. See Figure $S 9$ for a comparison of the SERS and Raman spectra of pATP.
We first tested the sensitivity of $\mathrm{ZrO}_{2}$-protected TERS probes for monitoring $\mathrm{pATP} \rightarrow \mathrm{DMAB}$ in air and aqueous environments. For measurements in air, we placed the TERS probe in contact with the Ag substrate and carried out Raman mapping the probe apex around. Figure 3a shows the map of the $1437 \mathrm{~cm}^{-1}$ DMAB Raman band intensity, in which a much stronger intensity is observed at the probe apex position. Comparison of average spectra measured at the TERS probe apex in Figure 3a and away from it (Figure S10) showed that the DMAB bands are visible only in the TERS spectra measured at the probe apex, indicating a strong LSPR. Furthermore, the TERS signal measured at the position of maximum intensity in Figure $3 \mathrm{a}$ was found to be $\sim 12 \times$ stronger than the SERS signal measured at the same position with the probe retracted from the sample, as shown in Figure $3 c$. To rule out the possibility of the zirconia-protected TERS probe interfering in the reaction at the sample, we conducted similar TERS measurements on a thin film of pATP mixed with PMMA spin-coated on a glass substrate (Figure S11). TERS spectra measured at the TERS probe apex on this sample did not show signal from any DMAB $a_{g}$ Raman bands, whereas the $a_{1}$ Raman bands of pATP at 1086 and $1591 \mathrm{~cm}^{-1}$ were enhanced by a contrast of 5.3 in the TERS near-field. This demonstrates the chemical inertness of $\mathrm{ZrO}_{2}$-protected TERS probes for investigation of this catalytic reaction.

Next, we examined the sensitivity of the $\mathrm{ZrO}_{2}$-protected TERS probes for monitoring pATP $\rightarrow$ DMAB in an aqueous environment. In this case, TERS measurements were performed inside of a water droplet placed on the sample surface. Interestingly, compared to the SERS measurements in air, a $210 \times$ stronger signal was observed in water, as shown in Figure S12. This is in contrast to previous reports where the far-field Raman signal of molecular SAMs on Au was found to decrease by a factor of $>3$ in a liquid environment compared to air due to laser focus aberrations. ${ }^{25,34}$

A similar loss of optical coupling in water was also observed in our TERS system during far-field Raman measurements (Figure S13). However, for the pATP SAM on the heterogeneous $\mathrm{Ag}$ substrate, we speculate that this effect could arise due to the red shift of the surface plasmon resonance of the SERS substrate in an aqueous environment, ${ }^{52}$ which can lead to better matching of the surface plasmon resonance wavelength with the excitation laser, resulting in higher DMAB formation as well as a much stronger SERS signal. ${ }^{53-56}$ However, we cannot rule out other phenomena such as surface molecular diffusion ${ }^{57,58}$ and molecular reorientation, ${ }^{59}$ which may be affected by the presence of an aqueous environment. A detailed understanding of this effect is beyond the scope of the present study and warrants a separate investigation, to be pursued as part of our future work. A map of the $1437 \mathrm{~cm}^{-1} \mathrm{DMAB}$ band intensity measured around the TERS probe apex is shown in Figure $3 \mathrm{~b}$. Once again, a significantly higher DMAB signal intensity is observed at the probe apex, indicating a strong LSPR. See Figure S14 for a comparison of average spectra measured at the probe apex in Figure $3 b$ and away from it. The intensity of the TERS signal in water is found to be $\sim 12 \times$ stronger compared to the SERS signal measured at the same location (Figure $3 \mathrm{~d}$ ), clearly showing that the $\mathrm{ZrO}_{2}$-protected TERS probes retain their plasmonic sensitivity in water.

Furthermore, comparison of average spectra measured at the probe apex and away from it in Figure S14 also confirms the chemical inertness rendered by $\mathrm{ZrO}_{2}$ protection. In the Raman 

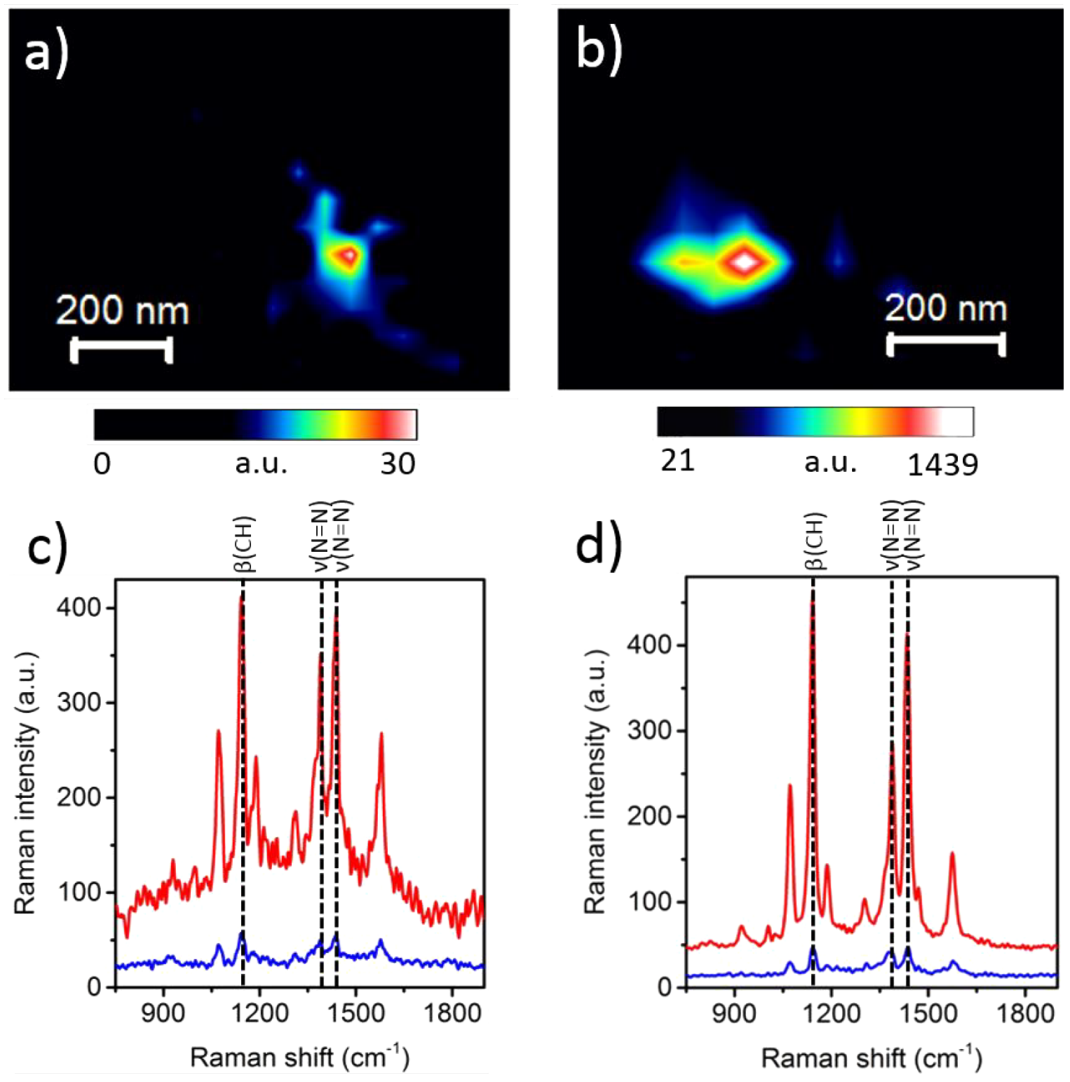

Figure 3. Maps of $\mathrm{pATP} \rightarrow \mathrm{DMAB}$ at the TERS probe apex obtained using the intensity of the $1437 \mathrm{~cm}^{-1}\left(\nu_{\mathrm{N}=\mathrm{N}}\right)$ DMAB Raman band measured from the pATP SAM on the Ag substrate in (a) air and (b) water. Integration time: $1 \mathrm{~s}$. Laser power: $117 \mu \mathrm{W}$. Pixel size: $50 \mathrm{~nm}$. TERS (red) and SERS (blue) spectra measured at the position of maximum DMAB signal in (c) Figure 3a and (d) Figure 3b, with the TERS probe in contact and retracted from the sample, respectively. Integration time: $60 \mathrm{~s}$ (Figure 3c) and $1 \mathrm{~s}$ (Figure 3d). The asymmetric shape of the reaction areas at the TERS probe apex shown in Figure 3 most likely arises from the combination of the random distribution of Ag grains at the TERS probe apex resulting from the thermal deposition of the Ag layer and the inhomogeneous and asymmetric distribution of the electromagnetic (EM) field intensity in the laser focal spot.

spectra, the $\nu_{\mathrm{C}-\mathrm{S}}$ vibrational mode at $\sim 1071 \mathrm{~cm}^{-1}$ is assigned to both pATP and DMAB, whereas the $\nu_{\mathrm{N}=\mathrm{N}}$ modes at 1390 and $1437 \mathrm{~cm}^{-1}$ and the $\beta_{\mathrm{C}-\mathrm{H}}$ mode at $1142 \mathrm{~cm}^{-1}$ are assigned exclusively to $\mathrm{DMAB} .{ }^{46}$ Therefore, the relative conversion of pATP to DMAB can be assessed using the ratio of the average intensity of DMAB bands $\left(I_{\mathrm{DMAB}}\right)$ to $I_{1071}$, removing the effect of fluctuations in the absolute TERS signal. ${ }^{60}$ Calculations of $I_{\mathrm{DMAB}} / I_{1071}$ at locations P1-P4 in Figure S14 are presented in Table $S 1$. At all four locations, $I_{\mathrm{DMAB}} / I_{1071}$ is found to be $\sim 1.9$, indicating that the conversion of pATP to $\mathrm{DMAB}$ is unaffected by the presence of the $\mathrm{ZrO}_{2}$-protected TERS probe, and it can therefore be considered as chemically noninterfering.

Finally, we performed nanoscale chemical imaging of this reaction on a Ag substrate in water. Figure 4a shows an AFM topography image of the surface, and Figure $4 \mathrm{~b}$ shows a TERS map of the pATP $\rightarrow$ DMAB conversion, obtained using the $I_{\mathrm{DMAB}} / I_{1071}$ ratio in an area of $500 \times 500 \mathrm{~nm}^{2}$. The conversion exhibits a high degree of spatial heterogeneity across the $\mathrm{Ag}$ substrate employed, and a histogram analysis of the $I_{\mathrm{DMAB}} / I_{1071}$ ratio across this map is consistent with a broad range of catalytic activities (Figure 4c). Furthermore, the TERS map in Figure $4 \mathrm{~b}$ exhibits a number of highly localized regions of notably high pATP $\rightarrow$ DMAB conversion. Some of these "reaction hotspots" are labeled in Figure $4 \mathrm{~b}$ (marked as 1-3), and corresponding spectra are shown in Figure $4 \mathrm{~d}$. For comparison, example spectra from regions of low conversion are also shown in Figure 4d and labeled as 4-6 in Figure 4b. The locations of the most active spots can be seen in the map presented in Figure S15a, in which the contrast has been adjusted to improve visualization, and additional example spectra are shown in Figure S15b. We propose that these extremely confined regions of high conversion reflect the plasmon-assisted nature of the pATP $\rightarrow$ DMAB reaction, ${ }^{51}$ in which LSPR at or in between individual Ag nanoparticles can confine light to a $<1 \mathrm{~nm}^{3}$ volume. ${ }^{61}$ We note that a high conversion of pATP to DMAB at a particular position on the Ag substrate could be caused by two factors: (1) strong LSPR coupling between the $\mathrm{ZrO}_{2}$-protected TERS probe apex and the Ag substrate or (2) LSPR within the SERS hotspots present on the Ag substrate. In the current study, it is not possible to distinguish between these two contributing factors as deconvoluting the influence of the TERS probe LSPR on the surface reaction remains a challenging problem beyond the scope of this work. Line profile analysis of the high activity regions (Figure S16 and Table S2) indicates that they are typically confined to one or two pixels (100-200 $\mathrm{nm}^{2}$ ), consistent with nanoscale spatial resolution. The stability of the $\mathrm{ZrO}_{2}$-protected TERS probe during TERS mapping was also assessed by comparing the first and last spectra recorded in the TERS map (see Figure S17), in which a similar signal intensity was observed.

In summary, in this work, we have addressed the key challenges of plasmonic degradation, chemical inertness, and 

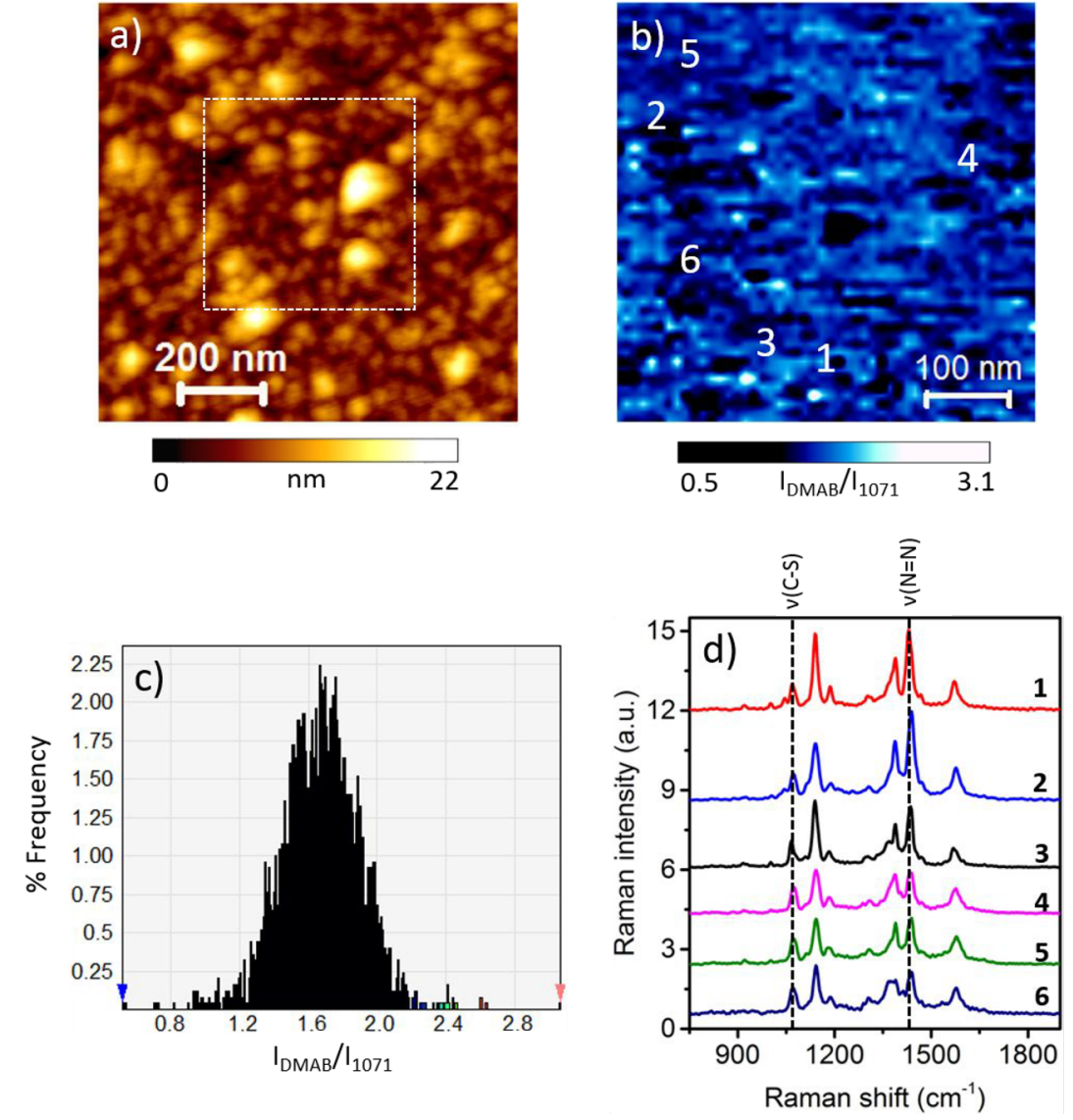

Figure 4. (a) AFM topography image of a heterogeneous Ag substrate functionalized with pATP. (b) TERS map of the $I_{\mathrm{DMAB}} / I_{1071}$ intensity ratio for the area marked in (a). Integration time: $1 \mathrm{~s}$. Laser power: $117 \mu \mathrm{W}$. Pixel size: $10 \mathrm{~nm}$. (c) Histogram showing the \% frequency of the $I_{\mathrm{DMAB}} / I_{1071}$ ratio in the pATP $\rightarrow$ DMAB TERS map in (b). (d) TERS spectra from the locations marked as $1-6$ in (b) showing different degrees of conversion across the TERS map. Spectra have been normalized to the intensity of the $1071 \mathrm{~cm}^{-1}$ band for comparison.

instability within a liquid environment of Ag-coated TERS probes by developing a novel method of protecting them with an ultrathin dielectric layer of zirconia. This successfully extends the lifetime of the probes over $850 \times$, from a few hours to $>4.5$ months, while rendering them chemically inert for the investigation of catalytic reactions in liquids at the nanoscale. Finally, using these robust and durable probes, we have demonstrated mapping of a model catalytic reaction on a heterogeneous substrate within a liquid environment with nanoscale spatial resolution. This work opens up the possibility of using TERS as a nanoanalytical tool to map molecular heterogeneities and interfacial dynamics in situ in diverse areas of scientific research such as heterogeneous catalyst systems, ${ }^{3}$ biology, ${ }^{62}$ and electrochemistry, ${ }^{63}$ in which nanoscale features at solid-liquid interfaces play a primary role in governing chemical behavior. Furthermore, this clear improvement in the probe lifetime and structural and chemical stability paves the way for using AFM-TERS for nondestructive, label-free, and nanoscale chemical characterization on a wide range of samples and environments.

\section{ASSOCIATED CONTENT}

\section{S Supporting Information}

The Supporting Information is available free of charge on the ACS Publications website at DOI: 10.1021/acs.jpclett.8b02496.

Experimental details of the TERS system, sample preparation, and preparation of TERS probes including $\mathrm{ZrO}_{2}$ coating; time series TERS measurements of unprotected Ag-coated TERS probes in air; time series TERS measurements of $\mathrm{ZrO}_{2}$-protected Ag-coated TERS probes in air; AFM topography of the Ag substrate; comparison of the Raman spectrum of bulk pATP and SERS spectrum of pATP adsorbed on a heterogeneous Ag substrate; analysis of pATP $\rightarrow$ DMAB at the apex of the Ag-coated TERS probe in air; chemical inertness test of the $\mathrm{ZrO}_{2}$-protected TERS probe; comparison of pATP $\rightarrow$ DMAB SERS spectra measured in air and water environments; comparison of far-field Raman spectra measured from a polystyrene thin film on glass in air and water; analysis of pATP $\rightarrow$ $\mathrm{DMAB}$ at the apex of the Ag-coated TERS probe in water; further analysis of the pATP $\rightarrow$ DMAB TERS map; and comparison of the first and last spectra measured in the TERS map (PDF)

\section{AUTHOR INFORMATION}

\section{Corresponding Author}

*E-mail: B.M.Weckhuysen@uu.nl.

ORCID

Naresh Kumar: 0000-0001-8953-5420

Andrew J. Wain: 0000-0002-8666-6158

Bert M. Weckhuysen: 0000-0001-5245-1426 


\section{Author Contributions}

${ }^{\S}$ N.K. and C.S.W. contributed equally to this work.

\section{Notes}

The authors declare no competing financial interest.

\section{ACKNOWLEDGMENTS}

B.M.W. acknowledges The Netherlands Organization for Scientific Research (NWO) for a Top research grant and the European Research Council (ERC) for an Advanced Grant (No. 321140). N.K. and A.J.W. acknowledge support from the National Measurement System of the UK Department of Business, Energy \& Industry Strategy.

\section{REFERENCES}

(1) Buurmans, I. L. C.; Weckhuysen, B. M. Heterogeneities of individual catalyst particles in space and time as monitored by spectroscopy. Nat. Chem. 2012, 4 (11), 873-886.

(2) Rothenberg, G. Catalysis: Concepts and Green Applications, 2nd ed.; Wiley-VCH: Weinheim, Germany, 2017.

(3) Weckhuysen, B. M. In situ Spectroscopy of Catalysts; American Scientific Publishers: Stevenson Ranch, 2004.

(4) Mestl, G. In situ Raman spectroscopy - a valuable tool to understand operating catalysts. J. Mol. Catal. A: Chem. 2000, 158 (1), $45-65$.

(5) Verma, P. Tip-enhanced Raman spectroscopy: Technique and recent advances. Chem. Rev. 2017, 117 (9), 6447-6466.

(6) Deckert-Gaudig, T.; Taguchi, A.; Kawata, S.; Deckert, V. Tipenhanced Raman spectroscopy-from early developments to recent advances. Chem. Soc. Rev. 2017, 46 (13), 4077-4110.

(7) Kumar, N.; Drozdz, M. M.; Jiang, H.; Santos, D. M.; Vaux, D. J. Nanoscale mapping of newly-synthesised phospholipid molecules in a biological cell using tip-enhanced Raman spectroscopy. Chem. Commun. 2017, 53 (16), 2451-2454.

(8) Yeo, B. S.; Amstad, E.; Schmid, T.; Stadler, J.; Zenobi, R. Nanoscale probing of a polymer-blend thin film with tip-enhanced Raman spectroscopy. Small 2009, 5 (8), 952-960.

(9) Lucia, A.; Cacioppo, O. A.; Iulianella, E.; Latessa, L.; Moccia, G.; Passeri, D.; Rossi, M. Capability of tip-enhanced Raman spectroscopy about nanoscale analysis of strained silicon for semiconductor devices production. Appl. Phys. Lett. 2017, 110 (10), 103105.

(10) Berweger, S.; Neacsu, C. C.; Mao, Y.; Zhou, H.; Wong, S. S.; Raschke, M. B. Optical nanocrystallography with tip-enhanced phonon Raman spectroscopy. Nat. Nanotechnol. 2009, 4 (8), 496499.

(11) Kumar, N.; Zoladek-Lemanczyk, A.; Guilbert, A. A.; Su, W.; Tuladhar, S. M.; Kirchartz, T.; Schroeder, B. C.; McCulloch, I.; Nelson, J.; Roy, D.; et al. Simultaneous topographical, electrical and optical microscopy of optoelectronic devices at the nanoscale. Nanoscale 2017, 9 (8), 2723-2731.

(12) Yano, T.-a.; Ichimura, T.; Kuwahara, S.; H’Dhili, F.; Uetsuki, K.; Okuno, Y.; Verma, P.; Kawata, S. Tip-enhanced nano-Raman analytical imaging of locally induced strain distribution in carbon nanotubes. Nat. Commun. 2013, 4, 2592.

(13) Su, W.; Kumar, N.; Dai, N.; Roy, D. Nanoscale mapping of intrinsic defects in single-layer graphene using tip-enhanced Raman spectroscopy. Chem. Commun. 2016, 52 (53), 8227-8230.

(14) Su, W.; Kumar, N.; Mignuzzi, S.; Crain, J.; Roy, D. Nanoscale mapping of excitonic processes in single-layer MoS2 using tipenhanced photoluminescence microscopy. Nanoscale 2016, 8 (20), 10564-10569.

(15) Su, W.; Kumar, N.; Krayev, A.; Chaigneau, M. In situ topographical chemical and electrical imaging of carboxyl graphene oxide at the nanoscale. Nat. Commun. 2018, 9 (1), 2891.

(16) Kumar, N.; Su, W.; Castro, F. A.; Weckhuysen, B. M. Tipenhanced Raman spectroscopy applications: From graphene to heterogeneous catalysis. Proc. SPIE Nanoscience \& Engineering VI 2018, 10726, 1072608 .
(17) Zhang, R.; Zhang, Y.; Dong, Z. C.; Jiang, S.; Zhang, C.; Chen, L. G.; Zhang, L.; Liao, Y.; Aizpurua, J.; Luo, Y.; Yang, J. L.; Hou, J. G. Chemical mapping of a single molecule by plasmon-enhanced Raman scattering. Nature 2013, 498 (7452), 82-86.

(18) Zrimsek, A. B.; Chiang, N.; Mattei, M.; Zaleski, S.; McAnally, M. O.; Chapman, C. T.; Henry, A.-I.; Schatz, G. C.; Van Duyne, R. P. Single-molecule chemistry with surface-and tip-enhanced Raman spectroscopy. Chem. Rev. 2017, 117 (11), 7583-7613.

(19) Kim, H.; Kosuda, K. M.; Van Duyne, R. P.; Stair, P. C. Resonance Raman and surface- and tip-enhanced Raman spectroscopy methods to study solid catalysts and heterogeneous catalytic reactions. Chem. Soc. Rev. 2010, 39 (12), 4820-4844.

(20) Hartman, T.; Wondergem, C. S.; Kumar, N.; van den Berg, A.; Weckhuysen, B. M. Surface-and tip-enhanced Raman spectroscopy in catalysis. J. Phys. Chem. Lett. 2016, 7 (8), 1570-1584.

(21) Domke, K. F.; Pettinger, B. In situ discrimination between axially complexed and ligand-free Co porphyrin on $\mathrm{Au}(111)$ with tipenhanced Raman spectroscopy. ChemPhysChem 2009, 10 (11), 1794-1798.

(22) van Schrojenstein Lantman, E. M.; Deckert-Gaudig, T.; Mank, A. J. G.; Deckert, V.; Weckhuysen, B. M. Catalytic processes monitored at the nanoscale with tip-enhanced Raman spectroscopy. Nat. Nanotechnol. 2012, 7 (9), 583-586.

(23) Kumar, N.; Stephanidis, B.; Zenobi, R.; Wain, A.; Roy, D. Nanoscale mapping of catalytic activity using tip-enhanced Raman spectroscopy. Nanoscale 2015, 7 (16), 7133-7137.

(24) Zhong, J.-H.; Jin, X.; Meng, L.; Wang, X.; Su, H.-S.; Yang, Z.-L.; Williams, C. T.; Ren, B. Probing the electronic and catalytic properties of a bimetallic surface with $3 \mathrm{~nm}$ resolution. Nat. Nanotechnol. 2016, 12 (2), 132-136.

(25) Martín Sabanés, N.; Driessen, L. M.; Domke, K. F. Versatile side-illumination geometry for tip-enhanced Raman spectroscopy at solid/liquid interfaces. Anal. Chem. 2016, 88 (14), 7108-7114.

(26) Martín Sabanés, N.; Ohto, T.; Andrienko, D.; Nagata, Y.; Domke, K. F. Electrochemical TERS elucidates potential-induced molecular reorientation of adenine $/ \mathrm{Au}(111)$. Angew. Chem., Int. Ed. 2017, 56 (33), 9796-9801.

(27) Mattei, M.; Kang, G.; Goubert, G.; Chulhai, D. V.; Schatz, G. C.; Jensen, L.; Van Duyne, R. P. Tip-enhanced Raman voltammetry: Coverage dependence and quantitative modeling. Nano Lett. 2017, 17 (1), 590-596.

(28) Kumar, N.; Kalirai, S.; Wain, A. J.; Weckhuysen, B. M. Nanoscale chemical imaging of a single catalyst particle with tipenhanced fluorescence microscopy. ChemCatChem 2019, 11 (1), 417-423.

(29) Schmid, T.; Yeo, B. S.; Leong, G.; Stadler, J.; Zenobi, R. Performing tip-enhanced Raman spectroscopy in liquids. J. Raman Spectrosc. 2009, 40 (10), 1392-1399.

(30) Scherger, J. D.; Foster, M. D. Tunable, liquid resistant tipenhanced Raman spectroscopy probes: Toward label-free nanoresolved imaging of biological systems. Langmuir 2017, 33 (31), $7818-7825$

(31) Touzalin, T.; Dauphin, A. L.; Joiret, S.; Lucas, I. T.; Maisonhaute, E. Tip-enhanced Raman spectroscopy imaging of opaque samples in organic liquid. Phys. Chem. Chem. Phys. 2016, 18 (23), 15510-15513.

(32) Zeng, Z.-C.; Huang, S.-C.; Wu, D.-Y.; Meng, L.-Y.; Li, M.-H.; Huang, T.-X.; Zhong, J.-H.; Wang, X.; Yang, Z.-L.; Ren, B. Electrochemical tip-enhanced Raman spectroscopy. J. Am. Chem. Soc. 2015, 137 (37), 11928-11931.

(33) Kurouski, D.; Mattei, M.; Van Duyne, R. P. Probing redox reactions at the nanoscale with electrochemical tip-enhanced Raman spectroscopy. Nano Lett. 2015, 15 (12), 7956-7962.

(34) Kumar, N.; Su, W.; Vesely, M.; Weckhuysen, B. M.; Pollard, A. J.; Wain, A. J. Nanoscale chemical imaging of solid-liquid interfaces using tip-enhanced Raman spectroscopy. Nanoscale 2018, 10 (4), $1815-1824$.

(35) Su, W.; Kumar, N.; Mignuzzi, S.; Crain, J.; Roy, D. Nanoscale mapping of excitonic processes in single-layer MoS 2 using tip- 
enhanced photoluminescence microscopy. Nanoscale 2016, 8 (20), 10564-10569.

(36) Kumar, N.; Spencer, S. J.; Imbraguglio, D.; Rossi, A. M.; Wain, A. J.; Weckhuysen, B. M.; Roy, D. Extending the plasmonic lifetime of tip-enhanced Raman spectroscopy probes. Phys. Chem. Chem. Phys. 2016, 18 (19), 13710-13716.

(37) Kalbacova, J.; Rodriguez, R. D.; Desale, V.; Schneider, M.; Amin, I.; Jordan, R.; Zahn, D. R. Chemical stability of plasmon-active silver tips for tip-enhanced Raman spectroscopy. Nanospectroscopy 2015, 1, 12-18.

(38) Opilik, L.; Dogan, Ü.; Szczerbiński, J.; Zenobi, R. Degradation of silver near-field optical probes and its electrochemical reversal. Appl. Phys. Lett. 2015, 107 (9), 091109.

(39) Yeo, B. S.; Stadler, J.; Schmid, T.; Zenobi, R.; Zhang, W. H. Tip-enhanced Raman spectroscopy - Its status, challenges and future directions. Chem. Phys. Lett. 2009, 472 (1-3), 1-13.

(40) Barrios, C. A.; Malkovskiy, A. V.; Kisliuk, A. M.; Sokolov, A. P.; Foster, M. D. Highly stable, protected plasmonic nanostructures for tip-enhanced Raman spectroscopy. J. Phys. Chem. C 2009, 113 (19), $8158-8161$.

(41) Opilik, L.; Dogan, U. z.; Li, C.-Y.; Stephanidis, B.; Li, J.-F.; Zenobi, R. Chemical production of thin protective coatings on optical nanotips for tip-enhanced Raman spectroscopy. J. Phys. Chem. C 2016, 120 (37), 20828-20832.

(42) Pourbaix, M. Atlas of Electrochemical Equilibria in Aqueous Solutions; National Association of Corrosion Engineers: Houston, TX, 1974.

(43) Huang, Y.-P.; et al. Shell-isolated tip-enhanced Raman and fluorescence spectroscopy. Angew. Chem., Int. Ed. 2018, 57 (25), $7523-7527$.

(44) Vaidya, P. D.; Lopez-Sanchez, J. A. Review of hydrogen production by catalytic aqueous-phase reforming. ChemistrySelect 2017, 2 (22), 6563-6576.

(45) Navarro, R. M.; Pena, M.; Fierro, J. Hydrogen production reactions from carbon feedstocks: fossil fuels and biomass. Chem. Rev. 2007, 107 (10), 3952-3991.

(46) Huang, Y.-F.; Zhu, H.-P.; Liu, G.-K.; Wu, D.-Y.; Ren, B.; Tian, Z.-Q. When the signal is not from the original molecule to be detected: chemical transformation of para-aminothiophenol on $\mathrm{Ag}$ during the SERS measurement. J. Am. Chem. Soc. 2010, 132 (27), 9244-9246.

(47) Mehtani, D.; Lee, N.; Hartschuh, R.; Kisliuk, A.; Foster, M.; Sokolov, A.; Cajko, F.; Tsukerman, I. Optical properties and enhancement factors of the tips for apertureless near-field optics. J. Opt. A: Pure Appl. Opt. 2006, 8 (4), S183-S190.

(48) Agapov, R. L.; Malkovskiy, A. V.; Sokolov, A. P.; Foster, M. D. Prolonged blinking with TERS probes. J. Phys. Chem. C 2011, 115 (18), 8900-8905.

(49) Kumar, N.; Rae, A.; Roy, D. Accurate measurement of enhancement factor in tip-enhanced Raman spectroscopy through elimination of far-field artefacts. Appl. Phys. Lett. 2014, 104 (12), 123106.

(50) Zhang, Z.; Merk, V.; Hermanns, A.; Unger, W. E. S.; Kneipp, J. Role of metal cations in plasmon-catalyzed oxidation: A case study of p-aminothiophenol dimerization. ACS Catal. 2017, 7 (11), 78037809.

(51) Zhao, L. B.; Zhang, M.; Huang, Y. F.; Williams, C. T.; Wu, D. Y.; Ren, B.; Tian, Z. Q. Theoretical study of plasmon-enhanced surface catalytic coupling reactions of aromatic amines and nitro compounds. J. Phys. Chem. Lett. 2014, 5 (7), 1259-1266.

(52) Mock, J. J.; Smith, D. R.; Schultz, S. Local refractive index dependence of plasmon resonance spectra from individual nanoparticles. Nano Lett. 2003, 3 (4), 485-491.

(53) Xu, P.; Kang, L.; Mack, N. H.; Schanze, K. S.; Han, X.; Wang, H.-L. Mechanistic understanding of surface plasmon assisted catalysis on a single particle: cyclic redox of 4-aminothiophenol. Sci. Rep. 2013, 3, 2997.

(54) Sun, M.; Xu, H. A novel application of plasmonics: Plasmondriven surface-catalyzed reactions. Small 2012, 8 (18), 2777-2786.
(55) Kang, L.; Xu, P.; Zhang, B.; Tsai, H.; Han, X.; Wang, H.-L. Laser wavelength- and power-dependent plasmon-driven chemical reactions monitored using single particle surface enhanced Raman spectroscopy. Chem. Commun. 2013, 49 (33), 3389-3391.

(56) Dai, Z. G.; Xiao, X. H.; Zhang, Y. P.; Ren, F.; Wu, W.; Zhang, S. F.; Zhou, J.; Mei, F.; Jiang, C. Z. In situ Raman scattering study on a controllable plasmon-driven surface catalysis reaction on $\mathrm{Ag}$ nanoparticle arrays. Nanotechnology 2012, 23 (33), 335701.

(57) Walder, R.; Nelson, N.; Schwartz, D. K. Single molecule observations of desorption-mediated diffusion at the solid-liquid interface. Phys. Rev. Lett. 2011, 107 (15), 156102.

(58) Nelson, N.; Walder, R.; Schwartz, D. K. Single molecule dynamics on hydrophobic self-assembled monolayers. Langmuir 2012, 28 (33), 12108-12113.

(59) Pardo, L.; Boland, T. A quantitative approach to studying structures and orientation at self-assembled monolayer/fluid interfaces. J. Colloid Interface Sci. 2003, 257 (1), 116-120.

(60) Bishnoi, S. W.; Rozell, C. J.; Levin, C. S.; Gheith, M. K.; Johnson, B. R.; Johnson, D. H.; Halas, N. J. All-optical nanoscale pH meter. Nano Lett. 2006, 6 (8), 1687-1692.

(61) Benz, F.; Schmidt, M. K.; Dreismann, A.; Chikkaraddy, R.; Zhang, Y.; Demetriadou, A.; Carnegie, C.; Ohadi, H.; de Nijs, B.; Esteban, R.; Aizpurua, J.; Baumberg, J. J. Single-molecule optomechanics in "picocavities. Science 2016, 354 (6313), 726-729.

(62) Dufrêne, Y. F. Using nanotechniques to explore microbial surfaces. Nat. Rev. Microbiol. 2004, 2 (6), 451-460.

(63) Weaver, M. J.; Gao, X. In situ electrochemical surface science. Annu. Rev. Phys. Chem. 1993, 44 (1), 459-494. 